\title{
Hyperfine Fields and Isomer Shifts in Magnetically Ordered Europium Compounds
}

\author{
H. H. WiCKMan, I. Nowik, AND J. H. Wernick \\ Bell Telephone Laboratories, Inc., Murray Hill, New Jersey \\ AND \\ D. A. SHIRLEY \\ Laurence Radiation Laboratory, University of California, Berkeley, California \\ AND \\ R. B. FRANKEL \\ National Magnet Laboratory, Cambridge, Massachusetts
}

\begin{abstract}
The Mössbauer technique was employed to study hyperfine fields in several ordered divalent europium compounds at low temperatures. The intermetallics $\mathrm{EuCu}_{2}, \mathrm{EuAl}_{2}$, and $\mathrm{EuAl}_{4}$ were ordered at helium temperatures and the observed fields and isomer shifts were $189(8) \mathrm{kOe},-0.88 \mathrm{~cm} / \mathrm{sec} ; 278(8) \mathrm{kOe},-0.97$ $\mathrm{cm} / \mathrm{sec}$; and $290(8) \mathrm{kOe},-1.14 \mathrm{~cm} / \mathrm{sec}$, respectively. Comparison of these results and previous measurements with EuO, EuS, and Eu metal indicates a possible correlation between isomer shift and internal field. With the exception of $\mathrm{EuCu}_{2}$ the internal fields increase with increasing magnitude of isomer shift. Interpretation of this trend with a core polarization model yields a value of $120 \pm 20 \mathrm{kOe}$ for the field per $6 s$ valence electron.
\end{abstract}

$\mathbf{S}$ EVERAL Mössbauer experiments have shown that divalent ${ }^{151} \mathrm{Eu}$ ions in different compounds display large and varied isomer shifts relative to an $\mathrm{Eu}_{2} \mathrm{O}_{3}$ source. ${ }^{1-4}$ In addition, the europium chalcogenides and several intermetallic systems are ordered at low temperatures. In this paper we report measurements of "saturation" hf interactions (hfs) and isomer shifts (IS) in a number of compounds and discuss the possibility of a correlation between these parameters. A core polarization model leading to a correlation of hfs and IS and previously applied to Eu metal, EuO, and $\mathrm{EuS}^{5}$ is also applicable to $\mathrm{EuAl}_{2}$ and $\mathrm{EuAl}_{4}$. However, such a simple picture is not applicable without modification to similar measurements in $\mathrm{EuCu}_{2}$. Recently, Winkler ${ }^{6}$ has also discussed core polarization in europium, and Danon and de Graaf $^{7}$ have noted an apparent correlation between isomer shifts and bulk magnetic properties.

Sources of ${ }^{151} \mathrm{Gd}$ or ${ }^{151} \mathrm{Sm}$ in oxide form were employed. Isomer shifts were determined at $77^{\circ} \mathrm{K}$; the magnetic systems were ordered at helium temperatures. The experimental data and other pertinent information are summarized in Table $\mathbf{I}$.

In divalent europium compounds the hyperfine fields arise from polarization of $s$ electrons (owing to the paramagnetic $4 f$ electrons) which yield a resultant field at the nucleus via the Fermi contact interaction. Isomer shifts in ${ }^{151} \mathrm{Eu}^{2+}$ are proportional to the difference

1 P. H. Barrett and D. A. Shirley, Phys. Rev. 131, 123 (1963).

2 P. Kienle, Rev. Mad. Phys. 36, 372 (1964).

3 P. Brix, S. Hüfner, P. Kienle, and D. Quitman, Phys. Letters 13, $140(1964)$.

${ }^{4}$ D. A. Shirley, R. B. Frankel, and H. H. Wickman, Rev. Mod. Phys. 36, 392 (1964). An obvious error occurred in this abstract: in line 12, "Divalent $\mathrm{Eu}^{2+}$ " should read "Trivalent $\mathrm{Eu}^{3+}$."

B H. H. Wickman, Ph.D. thesis, Berkeley (1964), unpublished.

- R. Winkler, Z. Physik 184, 433 (1965).

${ }^{7} \mathrm{~J}$. Danon and A. M. de Graaf (private communication). between electron densities at absorber nuclei and similar densities in source nuclei,

$$
\text { IS } \propto\left(|\psi(0)|_{\text {abs }^{2}}-|\psi(0)|_{\text {source }}^{2}\right) .
$$

Because both hfs and IS are functions of $|\psi(0)|^{2}$ it is reasonable to consider the possibility of a correlation between the two parameters.

For $\mathrm{Eu}^{2+}$ compounds we write the configuration as $4 f^{7} 5 s^{2} 6 s^{x}$ to emphasize the shielding (at the nucleus) of the outer $5 s$ electrons by $4 f$ electrons. ${ }^{8}$ In the experiments the source, $\mathrm{Eu}^{3+}, 4 f^{6} 5 s^{2} 6 s^{0}$, has larger $5 s$ electron density at the nucleus than $\mathrm{Eu}^{2+}, 4 f^{7} 5 s^{2} 6 s^{x} 4,5$; all the divalent compounds studied showed large negative IS. For example, in ionic $\mathrm{EuF}_{2}$ with $\mathrm{Eu}^{2+}, 4 f^{7} 5 s^{2} 6 s^{0}$, the shift was $-1.5 \mathrm{~cm} / \mathrm{sec}$; for intermediate compounds $4 f^{7} 5 s^{2} 6 s^{x}$ shifts with magnitudes less than $1.5 \mathrm{~cm} / \mathrm{sec}$ are expected as $0 \leq x \leq 2$. That is, as 6 s electrons (valence or conduction) are added, the charge density at the nucleus increases and | IS | decreases.

Consider now the spin density yielding a hyperfine field $H_{\text {eff }}$ which may be approximated as a constant "core" or non- $6 s$ contribution and a varying contribution from the $6 s$ electrons: $H_{\text {eff }}=H_{\text {core }}+H\left(6 s^{x}\right)$. Theoretical calculations indicate the two contributions to the field are oppositely directed. ${ }^{9}$ Thus, if the polarizability remains constant, $H_{\text {int }}$ must decrease as $x \rightarrow 2$. It is suggested below that in $\mathrm{EuCu}_{2}$ the polarizability is affected by the copper $3 d$ and $4 s$ bands and the assumption made above fails in such cases. However, the approximation of constant polarizabilities should be satisfied in the other systems studied. Hence we write

\footnotetext{
${ }^{8}$ L. R. Walker, G. K. Wertheim, and V. Jaccarino, Phys. Rev. Letters 6, 98 (1961).

R. E. Watson and A. J. Freeman, Phys. Rev. 123, 2027 (1961).
} 
TABLE I. Isomer shifts and magnetic interactions in several europous compounds.

\begin{tabular}{|c|c|c|c|c|}
\hline & $\begin{array}{c}\text { Transition } \\
\text { temperatures }\left({ }^{\circ} \mathrm{K}\right)\end{array}$ & $\begin{array}{c}-\mathrm{IS} \\
(\mathrm{cm} / \mathrm{sec})\end{array}$ & $\begin{array}{l}H_{\text {eff }}\left({ }^{\circ} \mathrm{K}\right) \\
\quad(\mathrm{kOe})\end{array}$ & $\begin{array}{c}\text { Other measurements } \\
\text { (of IS) }\end{array}$ \\
\hline $\mathrm{EuO}$ & $69\left(T_{c}\right)$ & $1.15(3)$ & $300 \pm 8(4.2)$ & \\
\hline EuS & $16.3\left(T_{c}\right)$ & $1.26(3)$ & $331 \pm 10(4.2)$ & 1.17(3) Ref. 3 \\
\hline EuSe & $7\left(T_{c}\right)$ & $1.26(3)$ & $331 \pm 10(4.2)$ & 1.22(2) Ref. 3 \\
\hline $\mathrm{EuF}_{2}$ & $2\left(T_{c}\right)$ & $1.50(3)$ & & \\
\hline $\mathrm{EuCu}_{2}$ & $20>T_{n}>4.2$ & $0.88(3)$ & $196 \pm 10(1.8)$ & \\
\hline $\mathrm{EuAl}_{2}$ & $77>T_{n}>20$ & $0.97(3)$ & $278 \pm 10(1.8)$ & \\
\hline $\mathrm{EuAl}_{4}$ & $\sim 6$ & $1.14(3)$ & $290 \pm 10(1.8)$ & \\
\hline Eu metal & $87\left(T_{n}\right)$ & $0.82(3)$ & $265 \pm 10(4.2)$ & \\
\hline
\end{tabular}

$H_{\text {int }}=H_{\text {core }}+\alpha x$ and for isomer shifts a similar expression, IS $=\mathrm{IS}_{\text {core }}+\alpha^{\prime} x$. These expressions combine to yield a linear relation $H_{\text {int }}=\beta \mathrm{IS}+\gamma$ between hfs and IS. In Fig. 1 this relation is tested by plotting $H_{\text {eff }}$ vs IS. The dotted line in Fig. 1 represents a linear approximation to the data and yields the constant $\beta$, i.e., the field per unit isomer shift which is directly proportional to the number of $6 s$ electrons, as shown below. Thus the field per $6 s$ valence or conduction electron is

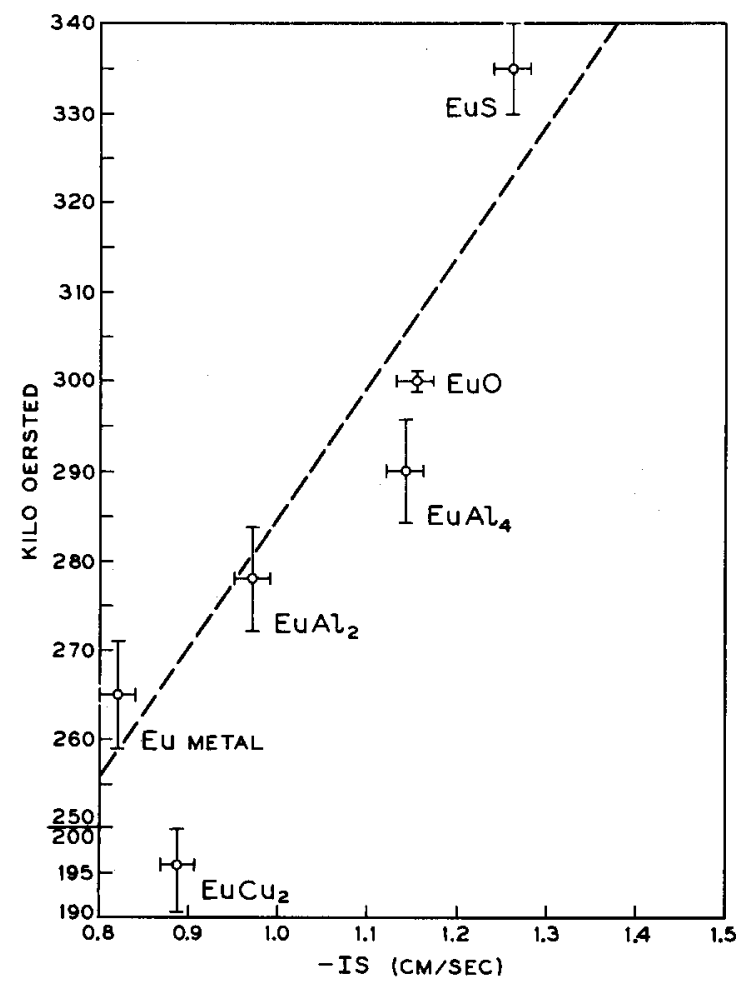

Fig. 1. Plot of $H_{\text {eff }}$ vs IS in ordered europium compounds. Note the different scale for $\mathrm{EuCu}_{2}$. found to be $120 \pm 20 \mathrm{kOe}$. This is in good agreement with $H_{\text {eff }}\left(\mathrm{EuAl}_{2}\right)-H_{\text {eff }}\left(\mathrm{GdAl}_{2}\right)=110 \mathrm{kOe}$, where the two compounds differ in one conduction electron. ${ }^{10}$ Comparing this field with that of an atomic europium $6 s$ electron we fine a polarization $2.5 \%$. Despite the approximate nature of the core polarization picture, the reasonably good correlation found here suggests the model has meaning. The obvious exception is $\mathrm{EuCu}_{2}$ where more complex interactions are evidently in effect.

Methfessel had discussed the many types of magnetic interaction possible in lanthanide systems. ${ }^{11}$ While the core contribution may be altered via indirect superexchange, it seems more likely that the interaction of the Eu $6 s$ electrons with $3 d$ and $4 s$ bands in copper destroys the constant polarizability assumption, which is more tenable with diamagnetic cations.

An immediate deduction from Fig. 1 is the "core" polarization field in the absence of $6 s$ electrons; this is $H_{\text {core }}=355 \pm 20 \mathrm{kOe}$, in agreement with the value 340 kOe obtained by Blumberg and Eisinger from ESR measurements with $\mathrm{Eu}^{2+} ; \mathrm{CaF}_{2}{ }^{12}$

Finally, we derive a relation between the $6 s$ character of europous compounds as a function of their chemical shift. The two constants in the equation IS $=\mathrm{IS}_{\text {oore }}+\alpha^{\prime} x$ may be estimated by using IS $=1.5 \mathrm{~cm} / \mathrm{sec}$, when $x=0$, and the relation between hf field and isomer shift in free europium atoms where $H_{\text {eff }}=70 \mathrm{kOe}$ and $x=2$, which implies IS $=0.4$ for the free atom. This method was used, for example, to estimate the number of $6 s$ valence electrons in Eu metal ${ }^{13}$ and more recently Brix et al. have used similar ideas to include other rare-earth ions. $^{3}$

\footnotetext{
${ }^{10}$ J. I. Budnick, R. E. Gegenwarth, and J. H. Wernick, Bull. Am. Phys. Soc. 10, 317 (1965).

${ }^{11}$ S. Methfessel, Z. Angew. Phys. 18, 414 (1965).

12 W. E. Blumberg and J. Eisinger, Bull. Am. Phys. Soc. 6, 141 (1961).
}

${ }^{13}$ D. A. Shirley, Rev. Mod. Phys. 36, 339 (1964). 